Article

\title{
Molecular Structures and Spectral Properties of Natural Indigo and Indirubin: Experimental and DFT Studies
}

\author{
Zixin Ju ${ }^{1}{ }^{1}$, Jie Sun ${ }^{2}$ and Yanping Liu ${ }^{1, *}$ \\ 1 Key Laboratory of Textile Science \& Technology of Ministry of Education, College of Textiles, \\ Donghua University, Shanghai 201620, China; irisju@126.com \\ 2 Shanghai Naturalism Biological Technology Co., Ltd., Shanghai 201616, China; simon@teamwisdom.com.cn \\ * Correspondence: liuyp@dhu.edu.cn; Tel.: +86-1376-496-1672
}

Academic Editors: Marcella Guiso and Ilaria Serafin

Received: 25 September 2019; Accepted: 21 October 2019; Published: 24 October 2019

\begin{abstract}
This paper presents a comparative study on natural indigo and indirubin in terms of molecular structures and spectral properties by using both computational and experimental methods. The spectral properties were analyzed with Fourier transform infrared (FTIR), Raman, UV-Visible, and fluorescence techniques. The density functional theory (DFT) method with B3LYP using 6-311G(d,p) basis set was utilized to obtain their optimized geometric structures and calculate the molecular electrostatic potential, frontier molecular orbitals, FTIR, and Raman spectra. The single-excitation configuration interaction (CIS), time-dependent density functional theory (TD-DFT), and polarization continuum model (PCM) were used to optimize the excited state structure and calculate the UV-Visible absorption and fluorescence spectra of the two molecules at B3LYP/6-311G(d,p) level. The results showed that all computational spectra agreed well with the experimental results. It was found that the same vibrational mode presents a lower frequency in indigo than that in indirubin. The frontier molecular orbital analysis demonstrated that the UV-Visible absorption and fluorescence bands of indigo and indirubin are mainly derived from $\pi \rightarrow \pi^{*}$ transition. The results also implied that the indigo molecule is more conjugated and planar than indirubin, thereby exhibiting a longer maximum absorption wavelength and stronger fluorescence peak.
\end{abstract}

Keywords: natural indigo; natural indirubin; FTIR; Raman; UV-Visible; fluorescence; DFT

\section{Introduction}

Natural indigo (C.I. 75780), mazarine powder extracted from the indigo plant, is one of the earliest and most popular vat dyestuffs [1,2]. Recently, the demand for natural indigo dye is increasing dramatically for its safety and biodegradability. Natural indigo is now being used by several fashion retail giants such as H\&M on a large scale to replace synthetic indigo dye for developing safe, green, and sustainable jeans. Since natural indigo originates from natural resources, it is treble the cost of synthetic indigo dye. Clear evidence of dyeing fabrics with natural indigo is needed to prevent adulteration and protect the interests of consumers [3]. Natural indigo is easily oxidized to indirubin during the extraction process, so the presence of indirubin and other unknown impurities is a key feature of natural indigo dye. In this regard, an in-depth understanding of similarities and differences in the molecular structures features and various vibrational spectra of indigo and indirubin molecules is critical for establishing the authenticity of natural indigo dye.

Indirubin (C.I. 75790), an isomer of indigo, which is an effective component of anti-leukemia agent, also has pharmacological effects such as sterilization. The difference in molecular structures of indigo and indirubin gives rise to different physical and chemical properties as well as their 
applications. As shown in Figure 1, indigo and indirubin with the molecular formula of $\mathrm{C}_{16} \mathrm{H}_{10} \mathrm{~N}_{2} \mathrm{O}_{2}$ have a molecular weight of 262.6 Daltons, both of which have the double indole structure. Indigo is a planar molecule with a symmetrical trans structure and a strong conjugation effect [4], in which two intramolecular hydrogen bonds are formed between the adjacent carbonyl groups and the imino groups. Indirubin is an asymmetric structural isomer of indigo by condensing two indole rings [5]. In their production processes, the conditions of reaction should be strictly controlled in order to avoid interconversion of indigo and indirubin [6].

(a)<smiles>O=C1/C(=C2\Nc3ccccc3C2=O)Nc2ccccc21</smiles>

(b)

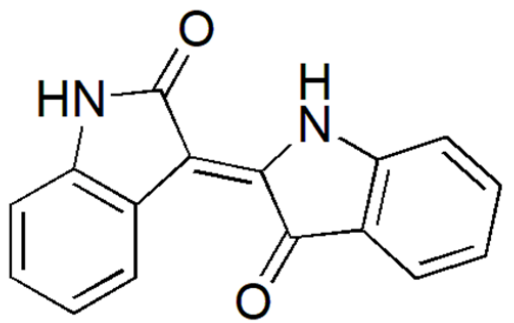

Figure 1. Chemical structures of indigo (a) and indirubin (b).

Previous works on indigo mainly focused on the dyeing process optimization and ancient textile identification [7-9]. In order to improve the dyeing performance of indigo and expand its application in electronics and other fields, considerable research efforts have been devoted to the DFT studies of indigo and its derivatives [10-14]. The earliest investigation on vibrational spectra of indigo has been conducted at the HF/3-21G level $[15,16]$. Later on, recalculations were carried out with more accurate methods or at larger basis sets, such as B3LYP/6-31G(d,p) [11], B3LYP/LANL2DZ/6-31G(d,p) [17], and B3LYP/6-311G $(\mathrm{d}, \mathrm{p})[18]$. A recent study confirmed the assignments of the main experimental features of the FTIR and Raman spectra below $1700 \mathrm{~cm}^{-1}$ by using B3LYP/6-311++G(d,p) [19]. Theoretical studies on the differences between the absorption of indigo molecule and its derivatives have been reported at TD-DFT/B3LYP/6-31G(d) [12], and their differences in emission spectra have also been investigated at the TD-DFT/B3LYP/6-31G $(\mathrm{d}, \mathrm{p})$ level [13]. The theoretical results indicated that the lowest excited singlet states of indigo and its derivatives were derived from the HOMO-LUMO transition. In addition to the studies based on a small basis set that neglected the solvent effect [12,13], the effects of environmental solvents and substitution patterns were assessed at TD-DFT/B3LYP/6-311+G(2d,p) [14]. It was found that indigo showed a strong bathochromic shift as the dielectric constant of the solvent increases due to the improved stability of the charged separation structure. The fluorescence quantum yield of polymeric indigo is an order of magnitude lower than that of indigo as a result of the energy transfer between different indigo chromophoric units [20].

The studies on indirubin were mainly related to the medical field, such as drug metabolism process and anti-tumor mechanism. In order to improve the efficacy and safety of indirubin, the influence of substituents on the geometry configuration and anti-cancer activity of indirubin has been studied computationally [21-24]. The optimized geometric structure and main vibrational modes assignment of indirubin were investigated at B3LYP/6-31G(d) level, but the vibrational spectra were not completely interpreted [22]. The frontier orbital study showed that the electrons in indirubin can jump easily due to the low HOMO-LUMO energy gap [23]. Computational study for the absorption spectra of indirubin by using hybrid methods B3LYP and PBE0 showed that the excitation responsible for the color typically corresponds to the HOMO $\rightarrow$ LUMO transition, which is a typical $\pi \rightarrow \pi^{*}$ transition with densities originating from the central double bond to the side single bonds [24].

Some efforts have already been made to investigate indigo, indirubin, and their derivatives computationally. However, the assignments of the whole vibrational spectral range of indirubin are still lacking. The excited state characterization of indirubin also has not been reported. To establish the authenticity of natural indigo dye, this work compared the molecular structural differences between 
indigo and indirubin from natural resources, comprehensively characterized their spectral properties, and analyzed the relationship between the luminescent mechanism, spectral characteristics, and molecular structures of the two molecules. Accordingly, the FTIR, Raman, UV-Visible, and fluorescence spectral properties of indigo and indirubin have been measured and calculated. It is expected that this study could provide a reference for the spectral analysis of indigo and indirubin and lay the scientific basis for the assessment of purity and identification of natural indigo dye.

\section{Results and Discussion}

\subsection{Geometry Optimization}

The computational optimized geometries of indigo and indirubin at B3LYP/6-311G(d,p) basis set are shown in Figure 2. The theoretical optimized structural parameters (bond distances and bond angles) were compared with the experimental data in the literature [4,25], and the results are listed in Table 1. The correlation coefficient $R^{2}$ for bond distances and angles of indigo are 0.95398 and 0.96445 , respectively. The corresponding values for bond distances and angles of indirubin amount to 0.8891 and 0.9660. Exemplary correlations are presented in Figure S1 (Supplementary Information). The slight deviations between the experimental and computational results can be attributed to the different phases, the computational system is only a single molecule, while the latter is a solid phase with inter-molecular interactions affect.

(a)

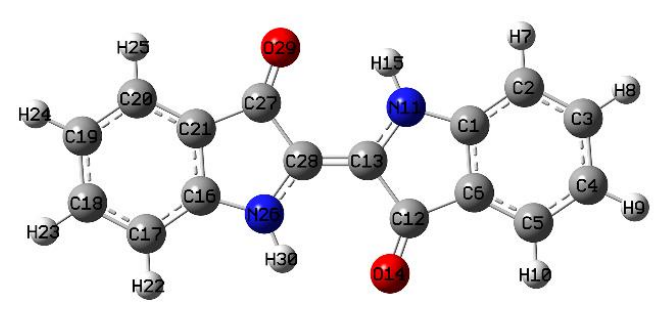

(b)

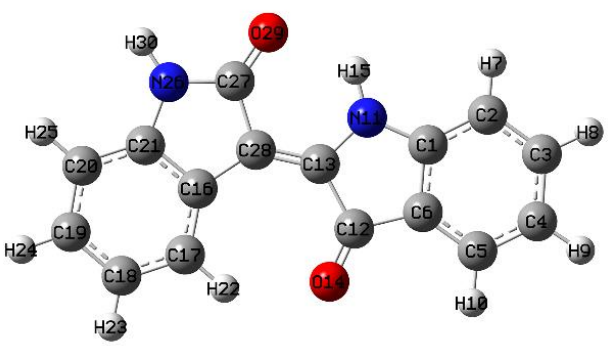

Figure 2. Optimized molecular structures of indigo (a) and indirubin (b) at B3LYP/6-311G(d,p).

Table 1. Optimized structural parameters for indigo and indirubin at B3LYP/6-311G(d,p).

\begin{tabular}{|c|c|c|c|c|c|}
\hline \multirow{2}{*}{$\begin{array}{c}\text { Structural Parameters } \\
\text { Internuclear Distance (̊̊) }\end{array}$} & \multicolumn{2}{|c|}{ Indigo } & \multirow{2}{*}{$\begin{array}{c}\text { Structural Parameters } \\
\text { Internuclear Distance (̊) }\end{array}$} & \multicolumn{2}{|c|}{ Indirubin } \\
\hline & Calc. & Exp. [5] & & Calc. & Exp. [25] \\
\hline$C(1)-C(2)$ & 1.393 & 1.393 & $\mathrm{C}(1)-\mathrm{C}(2)$ & 1.391 & 1.43 \\
\hline$C(2)-C(3)$ & 1.394 & 1.387 & $C(2)-C(3)$ & 1.396 & 1.41 \\
\hline $\mathrm{C}(3)-\mathrm{C}(4)$ & 1.403 & 1.386 & $C(3)-C(4)$ & 1.401 & 1.41 \\
\hline$C(4)-C(5)$ & 1.390 & 1.392 & $C(4)-C(5)$ & 1.392 & 1.41 \\
\hline$C(5)-C(6)$ & 1.393 & 1.387 & $C(5)-C(6)$ & 1.391 & 1.38 \\
\hline$C(1)-C(6)$ & 1.412 & 1.404 & $C(1)-C(6)$ & 1.405 & 1.39 \\
\hline$C(16)-C(17)$ & 1.393 & 1.393 & $C(16)-C(17)$ & 1.397 & 1.41 \\
\hline $\mathrm{C}(17)-\mathrm{C}(18)$ & 1.394 & 1.387 & $C(17)-C(18)$ & 1.396 & 1.42 \\
\hline $\mathrm{C}(18)-\mathrm{C}(19)$ & 1.403 & 1.386 & $\mathrm{C}(18)-\mathrm{C}(19)$ & 1.395 & 1.41 \\
\hline$C(19)-C(20)$ & 1.390 & 1.392 & $C(19)-C(20)$ & 1.389 & 1.41 \\
\hline$C(20)-C(21)$ & 1.393 & 1.387 & $C(20)-C(21)$ & 1.384 & 1.36 \\
\hline$C(21)-C(16)$ & 1.412 & 1.404 & $C(21)-C(16)$ & 1.417 & 1.40 \\
\hline$C(6)-C(12)$ & 1.468 & 1.424 & $C(6)-C(12)$ & 1.471 & 1.48 \\
\hline$C(12)-C(13)$ & 1.493 & 1.495 & $C(12)-C(13)$ & 1.524 & 1.53 \\
\hline$C(21)-C(27)$ & 1.468 & 1.424 & $C(16)-C(28)$ & 1.456 & 1.51 \\
\hline $\mathrm{C}(27)-\mathrm{C}(28)$ & 1.493 & 1.495 & $C(27)-C(28)$ & 1.496 & 1.48 \\
\hline $\mathrm{N}(11)-\mathrm{C}(13)$ & 1.379 & 1.382 & $\mathrm{~N}(11)-\mathrm{C}(13)$ & 1.374 & 1.40 \\
\hline $\mathrm{N}(26)-\mathrm{C}(28)$ & 1.379 & 1.382 & $\mathrm{~N}(26)-\mathrm{C}(27)$ & 1.377 & 1.38 \\
\hline $\mathrm{C}(1)-\mathrm{N}(11)$ & 1.387 & 1.380 & $\mathrm{C}(1)-\mathrm{N}(11)$ & 1.389 & 1.40 \\
\hline $\mathrm{C}(12)-\mathrm{O}(14)$ & 1.226 & 1.240 & $\mathrm{C}(12)-\mathrm{O}(14)$ & 1.216 & 1.21 \\
\hline$C(13)-C(28)$ & 1.358 & 1.342 & $C(13)-C(28)$ & 1.368 & 1.31 \\
\hline $\mathrm{C}(27)-\mathrm{O}(29)$ & 1.226 & 1.240 & $\mathrm{C}(27)-\mathrm{O}(29)$ & 1.226 & 1.25 \\
\hline
\end{tabular}


Table 1. Cont

\begin{tabular}{cccccc}
\hline Bond angle $\left(^{\circ}\right)$ & Calc. & Exp. [5] & Bond angle $\left(^{\circ}\right)$ & Calc. & Exp. [25] \\
\hline $\mathrm{C}(1)-\mathrm{N}(11)-\mathrm{C}(13)$ & 109.969 & 108.63 & $\mathrm{C}(1)-\mathrm{N}(11)-\mathrm{C}(13)$ & 111.654 & 110 \\
$\mathrm{C}(6)-\mathrm{C}(12)-\mathrm{O}(14)$ & 130.765 & 129.13 & $\mathrm{C}(6)-\mathrm{C}(12)-\mathrm{O}(14)$ & 128.603 & 128 \\
$\mathrm{C}(1)-\mathrm{N}(11)-\mathrm{H}(15)$ & 127.957 & 130.30 & $\mathrm{C}(1)-\mathrm{N}(11)-\mathrm{H}(15)$ & 128.015 & - \\
$\mathrm{C}(1)-\mathrm{C}(6)-\mathrm{C}(12)$ & 107.676 & 106.03 & $\mathrm{C}(1)-\mathrm{C}(6)-\mathrm{C}(12)$ & 107.884 & 106 \\
$\mathrm{C}(6)-\mathrm{C}(12)-\mathrm{C}(13)$ & 104.199 & 107.23 & $\mathrm{C}(6)-\mathrm{C}(12)-\mathrm{C}(13)$ & 104.402 & 106 \\
$\mathrm{C}(12)-\mathrm{C}(13)-\mathrm{N}(11)$ & 108.355 & 107.23 & $\mathrm{C}(12)-\mathrm{C}(13)-\mathrm{N}(11)$ & 106.487 & 106 \\
$\mathrm{C}(6)-\mathrm{C}(1)-\mathrm{N}(11)$ & 109.800 & 110.83 & $\mathrm{C}(6)-\mathrm{C}(1)-\mathrm{N}(11)$ & 109.573 & 112 \\
$\mathrm{C}(16)-\mathrm{C}(21)-\mathrm{C}(27)$ & 107.676 & 106.03 & $\mathrm{C}(16)-\mathrm{C}(21)-\mathrm{N}(26)$ & 109.065 & 110 \\
$\mathrm{C}(21)-\mathrm{C}(27)-\mathrm{C}(28)$ & 104.199 & 107.23 & $\mathrm{~N}(26)-\mathrm{C}(27)-\mathrm{C}(28)$ & 105.995 & 108 \\
$\mathrm{C}(27)-\mathrm{C}(28)-\mathrm{N}(26)$ & 108.355 & 107.23 & $\mathrm{C}(27)-\mathrm{C}(28)-\mathrm{C}(16)$ & 106.622 & 105 \\
$\mathrm{C}(16)-\mathrm{N}(26)-\mathrm{C}(28)$ & 109.969 & 108.63 & $\mathrm{C}(21)-\mathrm{N}(26)-\mathrm{C}(27)$ & 111.544 & 111 \\
$\mathrm{C}(21)-\mathrm{C}(16)-\mathrm{N}(26)$ & 109.800 & 110.83 & $\mathrm{C}(21)-\mathrm{C}(16)-\mathrm{N}(28)$ & 106.774 & 107 \\
$\mathrm{~N}(11)-\mathrm{C}(13)-\mathrm{C}(28)$ & 125.977 & 124.93 & $\mathrm{~N}(11)-\mathrm{C}(13)-\mathrm{C}(28)$ & 123.244 & 122 \\
$\mathrm{C}(21)-\mathrm{C}(27)-\mathrm{O}(29)$ & 130.765 & 129.13 & $\mathrm{~N}(26)-\mathrm{C}(27)-\mathrm{O}(29)$ & 125.564 & 126 \\
$\mathrm{C}(16)-\mathrm{N}(26)-\mathrm{H}(30)$ & 127.957 & 130.30 & $\mathrm{C}(21)-\mathrm{N}(26)-\mathrm{H}(30)$ & 126.055 & - \\
\hline
\end{tabular}

The data in Table 1 indicate that the $\mathrm{C}-\mathrm{C}$ bond lengths of the benzene rings in indigo and indirubin are, respectively, ranging from 1.386 to $1.412 \AA$ and from 1.360 to $1.430 \AA$, which are close to the $C-C$ bond length $(1.4 \AA)$ of the benzene molecule, showing a conjugation effect. The $\mathrm{C}-\mathrm{C}$ bond lengths of the heterocyclic rings in indigo and indirubin are 1.424-1.495 $\AA$ and $1.456-1.530 \AA$, respectively. The two bond length ranges are just in between those of the $C-C$ single bond ( $1.540 \AA$ ) and the $C=C$ double bond $(1.340 \AA)$, so the $\mathrm{C}-\mathrm{C}$ bonds were involved in the overall conjugation of the two molecules. The $\mathrm{N}(11)-\mathrm{C}(13)$ and $\mathrm{N}(26)-\mathrm{C}(28)$ bond lengths of the heterocyclic ring in indigo are identical being $1.379 \AA$ (1.382 $\AA$ in Exp.). The $\mathrm{N}(11)-\mathrm{C}(13)$ and $\mathrm{N}(26)-\mathrm{C}(27)$ bond lengths of the heterocyclic ring in indirubin are respectively $1.374 \AA$ and $1.377 \AA$ (1.40 $\AA$ and $1.38 \AA$ in Exp.), which are within the lengths of the normal $\mathrm{C}-\mathrm{N}$ single bond $(1.480 \AA)$ and $\mathrm{C}=\mathrm{N}$ double bond $(1.270 \AA)$. Consequently, the $\mathrm{C}-\mathrm{N}$ bonds in the two molecules are both delocalized bonds, which also participate in the overall conjugation of molecules.

Although there are many delocalized bonds both in the indigo and indirubin molecules, their conjugated degrees are different from each other. Different from the planar molecular indigo, the study on the X-ray crystal structure of indirubin has been reported that presumably due to the stereochemical constraints, and there is a small deviation $\left(4^{\circ}\right)$ from planarity between the two indole rings in indirubin $[5,25]$. This indicates that there is only one intramolecular hydrogen bond in indirubin between the $\mathrm{N}-\mathrm{H}$ and $\mathrm{C}=\mathrm{O}$ group while two in indigo. Therefore, the conjugation effect of the indirubin molecule is relatively weaker than that of the indigo molecule, and indigo is more conjugated and planar than indirubin. In addition, indigo is a nonpolar molecule with central symmetry and belongs to the $C_{2 h}$ point group. On the contrary, indirubin is a polar molecule and belongs to the $C_{s}$ point group.

\subsection{Analysis of Molecular Electrostatic Potential}

The molecular electrostatic potential surface (ESP) implying the interaction between molecules presents the distribution of electrostatic potential (electron + nucleus). The color in the ESP surface is a measure of the electrostatic potential value. Red represents electron-rich and partially negative charge, blue represents electron deficient and partially positive charge, light blue represents slightly electron deficient region, yellow represents slightly electron rich region, and green represents neutral.

Figure 3 shows the total electron density mapped with the electrostatic potential surface of indigo (a) and indirubin (b). The regions around the two oxygen atoms linking with the carbon atom by the double bond both in indigo and indirubin represent the most negative potential region (red), whereas the two hydrogen atoms linking with the nitrogen atoms have the largest positive charge (blue). From the ESP surface, it is confirmed that the negatively charged oxygen atoms tend to form hydrogen bonds with the positively charged hydrogen in indigo and indirubin. 
(a)

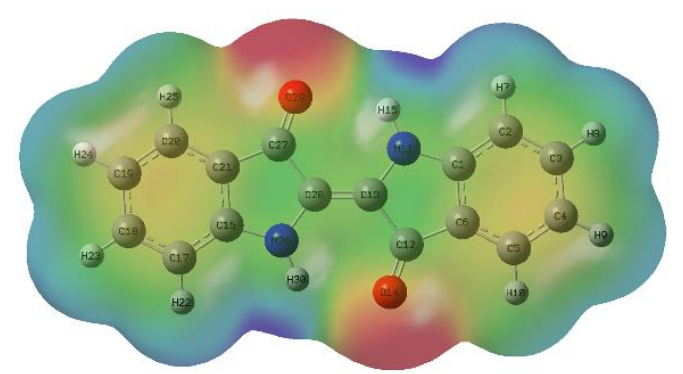

(b)

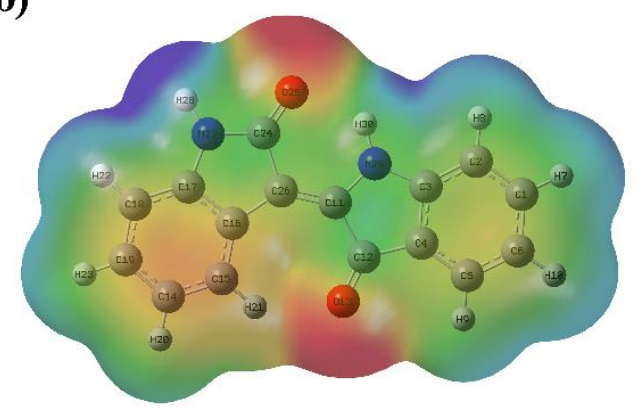

Figure 3. Total electron density mapped with electrostatic potential surface of indigo (a) and indirubin (b).

\subsection{Frontier Molecular Orbitals}

The frontier orbital theory holds that the energy gap between the highest occupied molecular orbital (HOMO) and the lowest unoccupied molecular orbital (LUMO) reflects the ability of electrons to transit from orbital to empty orbital, which to some extent represents the chemical activity of molecules. A molecule with a bigger energy gap is more inert and the electron transition is more difficult.

The energy values of $\mathrm{HOMO}$, LUMO, $\mathrm{HOMO}-1, \mathrm{LUMO}+1$ molecular orbitals, and the corresponding HOMO-LUMO energy gap of indigo and indirubin were computed at the theoretical level of B3LYP/6-311G $(\mathrm{d}, \mathrm{p})$. The wave function Multiwfn 3.6 combined with the visualization software VMD 1.9.3 was used to draw the frontier molecular orbits of indigo and indirubin as shown in Figure $4[26,27]$. As shown in Table 2, the HOMO-LUMO energy gap values of indigo and indirubin are $2.5035 \mathrm{eV}$ and $2.7511 \mathrm{eV}$, respectively. In this connection, indirubin of a higher HOMO-LUMO energy gap possesses better chemical stability.

(a)

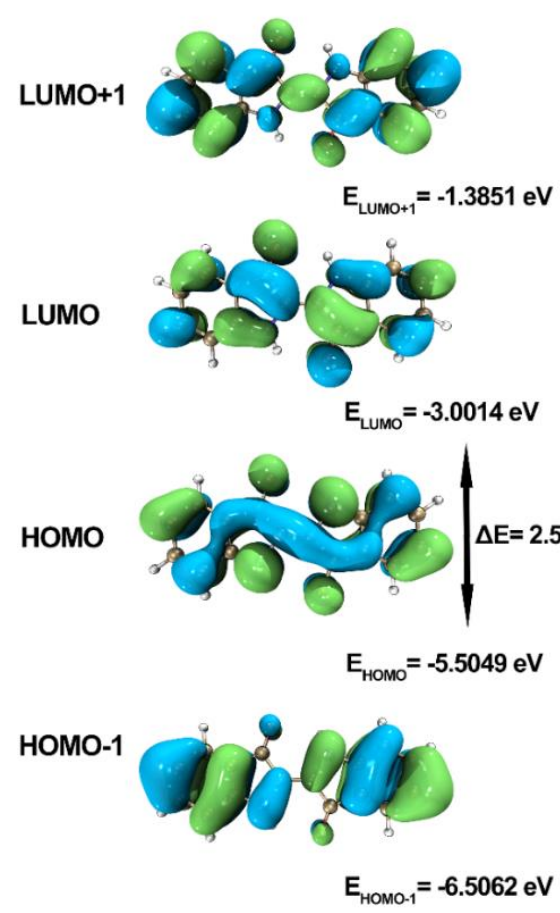

(b)
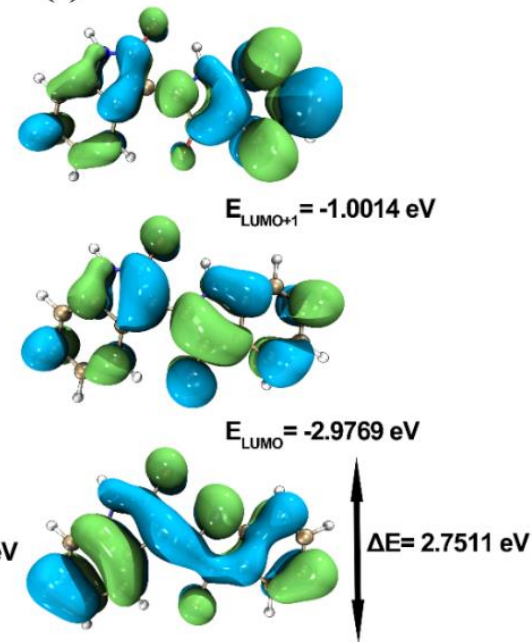

$E_{\text {номо }}=-5.7280 \mathrm{eV}$

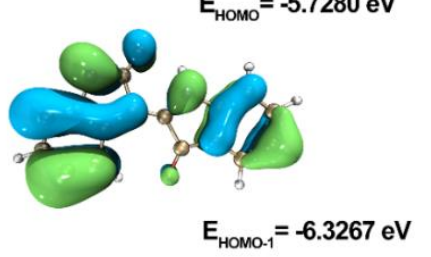

Figure 4. Frontier molecular orbitals of indigo (a) and indirubin (b). 
Table 2. Total molecular energy and frontier orbital energy.

\begin{tabular}{ccc}
\hline Thermodynamic Parameters (298 K) & Indigo & Indirubin \\
\hline $\mathrm{E}_{\mathrm{LUMO}+1}(\mathrm{eV})$ & -1.3851 & -1.0014 \\
$\mathrm{E}_{\mathrm{LUMO}}(\mathrm{eV})$ & -3.0014 & -2.9769 \\
$\mathrm{E}_{\mathrm{HOMO}}(\mathrm{eV})$ & -5.5049 & -5.7280 \\
$\mathrm{E}_{\mathrm{HOMO}-1}(\mathrm{eV})$ & -6.5062 & -6.3267 \\
$\Delta \mathrm{E}_{\mathrm{LUMO}-\mathrm{HOMO}}(\mathrm{eV})$ & 2.5035 & 2.7511 \\
\hline
\end{tabular}

\subsection{Vibrational Analysis}

Vibrational spectra including FTIR and Raman are valuable for elaborating the structures and compositional characteristics of materials. In order to interpret experimental spectra accurately, it is of great importance to predict the wavenumbers and spectral intensities of the molecule and identify vibration modes with quantum chemical computations [28]. The experimental FTIR and Raman spectra of indigo and indirubin are presented in Figure 5a,b, respectively. The Raman spectra were obtained only in the range of $100-1800 \mathrm{~cm}^{-1}$ due to the interference of their own fluorescence. Both experimental and computational vibrational wavelengths and assignments for Indigo and Indirubin at B3LYP/6-311G(d,p) basis set are detailed in Table 3, in which the vibrational modes of indigo and indirubin were identified by using Gauss View 6 . There is no virtual frequency in the computational results indicates that the optimized configuration is indeed a minimum point on the potential energy surface and the compound can exist stably. All computational wavelengths had been scaled by 0.967 to correct the theoretical error in this work.

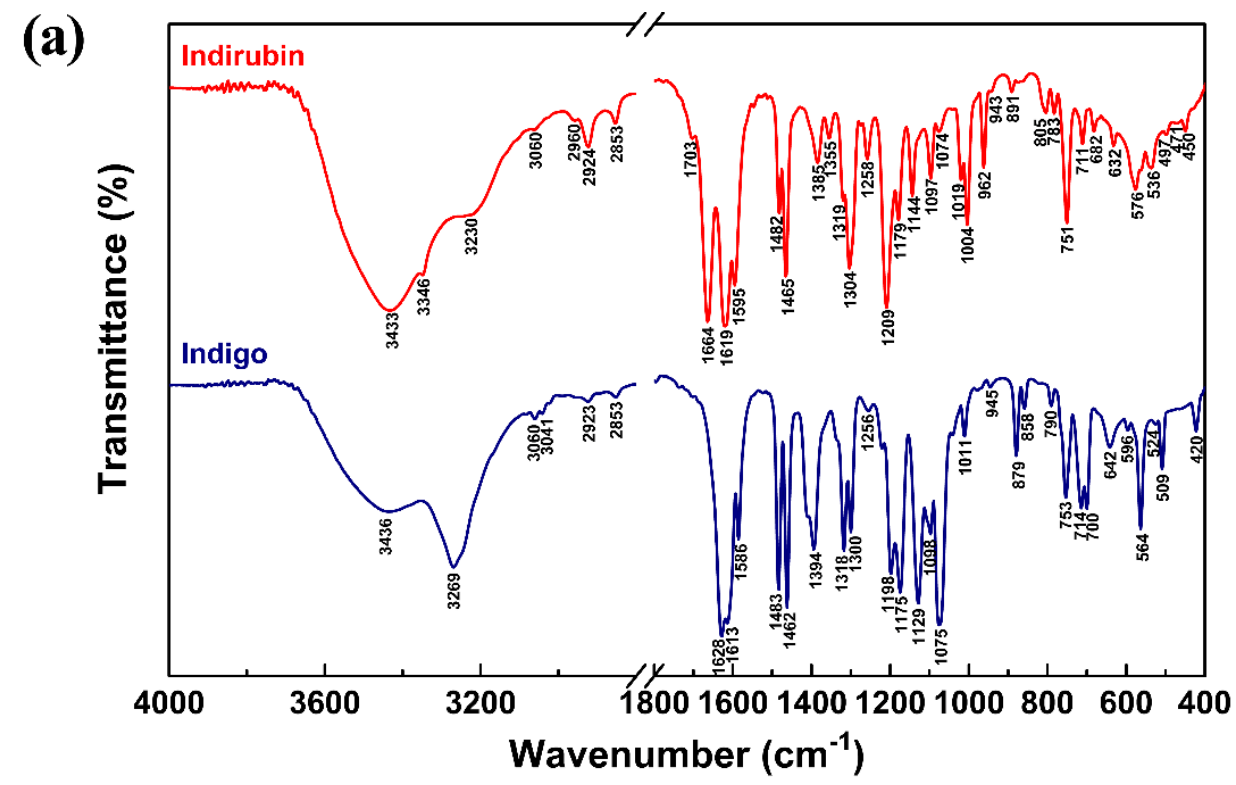

Figure 5. Cont. 


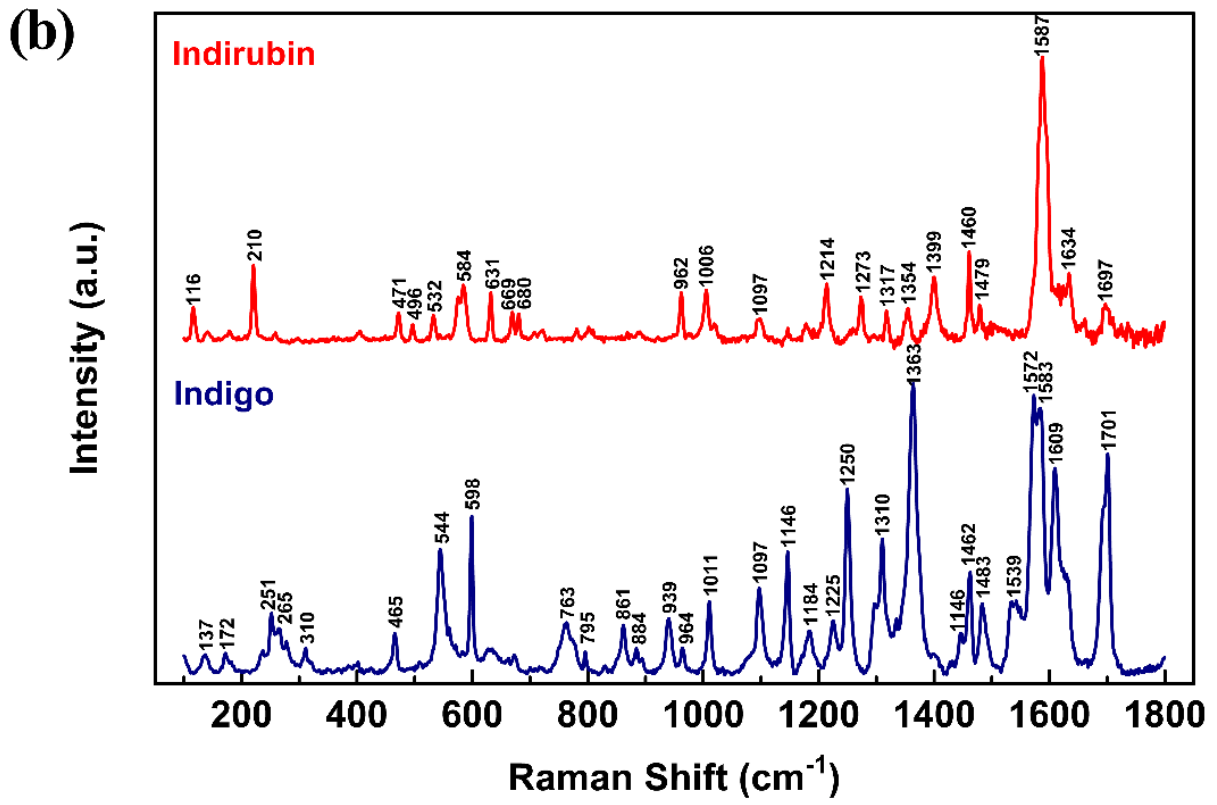

Figure 5. Infrared spectra (a) and Raman spectra (b) of indigo and indirubin.

Table 3. Experimental and computational vibrational wavelengths and assignments for indigo and indirubin at B3LYP/6-311G(d,p).

\begin{tabular}{|c|c|c|c|c|c|c|c|c|}
\hline \multirow{3}{*}{ Mode } & \multicolumn{4}{|c|}{ Indigo } & \multicolumn{4}{|c|}{ Indirubin } \\
\hline & \multicolumn{2}{|c|}{ Experimental } & \multirow{2}{*}{$\begin{array}{c}\text { Scaled } \\
\text { Computational }\end{array}$} & \multirow{2}{*}{$\begin{array}{c}\text { Tentative } \\
\text { Assignment }{ }^{1}\end{array}$} & \multicolumn{2}{|c|}{ Experimental } & \multirow{2}{*}{$\begin{array}{c}\text { Scaled } \\
\text { Computational }\end{array}$} & \multirow{2}{*}{$\begin{array}{c}\text { Tentative } \\
\text { Assignment }{ }^{1}\end{array}$} \\
\hline & IR & Raman & & & IR & Raman & & \\
\hline 1 & - & - & 3487.34 & $v_{\mathrm{S}} \mathrm{N}-\mathrm{H}$ & 3433 & - & 3530.01 & $v \mathrm{~N}-\mathrm{H}$ \\
\hline 2 & 3436 & - & 3486.36 & $v_{\text {as }} \mathrm{N}-\mathrm{H}$ & 3346 & - & 3398.59 & $v \mathrm{~N}-\mathrm{H}$ \\
\hline 3 & - & - & 3091.68 & $v_{\mathrm{s}} \mathrm{C}-\mathrm{H}$ & 3230 & - & 3124.20 & $v \mathrm{C}-\mathrm{H}$ \\
\hline 4 & 3269 & - & 3091.54 & $v_{\text {as }} \mathrm{C}-\mathrm{H}$ & 3060 & - & 3091.65 & $v \mathrm{C}-\mathrm{H}$ \\
\hline 5 & 3060 & - & 3082.39 & $v_{\text {as }} \mathrm{C}-\mathrm{H}$ & 2960 & - & 3084.44 & $v \mathrm{C}-\mathrm{H}$ \\
\hline 6 & 3041 & - & 3082.38 & $v \mathrm{C}-\mathrm{H}$ & 2924 & - & 3083.39 & $v \mathrm{C}-\mathrm{H}$ \\
\hline 7 & - & - & 3076.08 & $v_{\mathrm{s}} \mathrm{C}-\mathrm{H}$ & - & - & 3076.65 & $v \mathrm{C}-\mathrm{H}$ \\
\hline 8 & 2923 & - & 3076.07 & $v_{\text {as }} \mathrm{C}-\mathrm{H}$ & 2853 & - & 3073.11 & $v \mathrm{C}-\mathrm{H}$ \\
\hline 9 & 2853 & - & 3063.62 & $v_{\text {as }} \mathrm{C}-\mathrm{H}$ & - & - & 3064.40 & $v \mathrm{C}-\mathrm{H}$ \\
\hline 10 & - & - & 3063.60 & $v_{\mathrm{S}} \mathrm{C}-\mathrm{H}$ & - & - & 3061.94 & $v \mathrm{C}-\mathrm{H}$ \\
\hline 11 & - & 1701 & 1707.32 & $v \mathrm{C}=\mathrm{C}+v \mathrm{C}=\mathrm{O}$ & 1703 & - & 1710.64 & $v_{\mathrm{s}} \mathrm{C}=\mathrm{O}+v \mathrm{C}=\mathrm{C}$ \\
\hline 12 & 1628 & - & 1654.71 & $v \mathrm{C}=\mathrm{O}+\delta \mathrm{N}-\mathrm{H}$ & 1664 & 1697 & 1686.27 & $v_{\text {as }} \mathrm{C}=\mathrm{O}+\delta \mathrm{N}-\mathrm{H}$ \\
\hline 13 & - & 1609 & 1626.18 & $v \mathrm{C}=\mathrm{C}+v \mathrm{C}=\mathrm{O}+v \mathrm{C}-\mathrm{C}$ & - & - & 1611.54 & $v \mathrm{C}=\mathrm{C}+v \mathrm{C}-\mathrm{C}+\delta \mathrm{C}-\mathrm{H}$ \\
\hline 14 & 1613 & - & 1597.98 & $v \mathrm{C}-\mathrm{C}_{\text {ring }}+\delta \mathrm{C}-\mathrm{H}$ & 1619 & 1634 & 1603.14 & $v \mathrm{C}-\mathrm{C}+\delta \mathrm{C}-\mathrm{H}$ \\
\hline 15 & - & 1583 & 1590.48 & $v \mathrm{C}=\mathrm{C}+v \mathrm{C}=\mathrm{O}+v \mathrm{C}-\mathrm{C}$ & - & - & 1582.29 & $v \mathrm{C}-\mathrm{C}+\delta \mathrm{N}-\mathrm{H}+\delta \mathrm{C}-\mathrm{H}$ \\
\hline 16 & - & 1572 & 1572.14 & $v \mathrm{C}-\mathrm{C}_{\text {ring }}+\delta \mathrm{N}-\mathrm{H}$ & 1595 & 1587 & 1578.62 & $v \mathrm{C}-\mathrm{C}+v \mathrm{C}=\mathrm{C}+\delta \mathrm{N}-\mathrm{H}$ \\
\hline 17 & 1586 & - & 1571.73 & $v \mathrm{C}-\mathrm{C}_{\text {ring }}$ & - & - & 1566.60 & $v \mathrm{C}-\mathrm{C}+\delta \mathrm{C}-\mathrm{H}$ \\
\hline 18 & 1483 & - & 1468.51 & $v \mathrm{C}-\mathrm{C}+\delta \mathrm{C}-\mathrm{H}+\delta \mathrm{N}-\mathrm{H}$ & 1482 & 1479 & 1466.72 & $v \mathrm{C}-\mathrm{C}+\delta \mathrm{C}-\mathrm{H}+v \mathrm{~N}-\mathrm{H}$ \\
\hline 19 & - & 1483 & 1467.51 & $v \mathrm{C}-\mathrm{C}+\delta \mathrm{C}-\mathrm{H}+\delta \mathrm{N}-\mathrm{H}$ & - & - & 1461.41 & $v \mathrm{C}-\mathrm{C}+\delta \mathrm{C}-\mathrm{H}+v \mathrm{~N}-\mathrm{H}$ \\
\hline 20 & - & 1462 & 1441.41 & $v \mathrm{C}-\mathrm{C}_{\text {ring }}+\delta \mathrm{C}-\mathrm{H}$ & 1465 & 1460 & 1447.83 & $\delta \mathrm{C}-\mathrm{H}+v \mathrm{C}-\mathrm{C}$ \\
\hline 21 & 1462 & - & 1439.48 & $v \mathrm{C}-\mathrm{C}_{\text {ring }}+\delta \mathrm{C}-\mathrm{H}$ & - & - & 1442.37 & $v \mathrm{C}-\mathrm{C}+\delta \mathrm{C}-\mathrm{H}$ \\
\hline 22 & 1394 & - & 1399.78 & $\delta \mathrm{N}-\mathrm{H}+\delta \mathrm{C}-\mathrm{H}$ & 1385 & 1399 & 1390.31 & $\delta \mathrm{N}-\mathrm{H}+\delta \mathrm{C}-\mathrm{H}+\nu \mathrm{C}-\mathrm{C}$ \\
\hline 23 & - & 1363 & 1361.05 & $\delta \mathrm{N}-\mathrm{H}+\delta \mathrm{C}-\mathrm{H}$ & 1355 & 1354 & 1364.94 & $\delta \mathrm{N}-\mathrm{H}+\delta \mathrm{C}-\mathrm{H}+v \mathrm{C}-\mathrm{N}$ \\
\hline 24 & - & & 1340.83 & $v \mathrm{C}-\mathrm{C}+v \mathrm{C}-\mathrm{N}+\delta \mathrm{C}-\mathrm{H}$ & - & - & 1324.29 & $v \mathrm{C}-\mathrm{C}+v \mathrm{C}-\mathrm{N}+\delta \mathrm{C}-\mathrm{H}$ \\
\hline 25 & 1318 & - & 1310.37 & $v \mathrm{C}-\mathrm{C}_{\text {ring }}+\delta \mathrm{C}-\mathrm{H}$ & - & 1317 & 1308.36 & $v \mathrm{C}-\mathrm{C}+\delta \mathrm{C}-\mathrm{H}$ \\
\hline 26 & - & 1310 & 1299.01 & $\delta \mathrm{C}-\mathrm{H}+v \mathrm{C}-\mathrm{C}_{\text {ring }}$ & 1319 & - & 1276.29 & $\delta \mathrm{C}-\mathrm{H}+v \mathrm{C}-\mathrm{N}+v \mathrm{C}-\mathrm{C}$ \\
\hline 27 & 1300 & - & 1276.19 & $\delta \mathrm{C}-\mathrm{H}$ & 1304 & 1273 & 1272.39 & $\delta \mathrm{C}-\mathrm{H}+\delta \mathrm{N}-\mathrm{H}+v \mathrm{C}-\mathrm{C}$ \\
\hline 28 & 1256 & - & 1236.96 & $v \mathrm{C}-\mathrm{N}+v \mathrm{C}-\mathrm{C}+\delta \mathrm{C}-\mathrm{H}$ & - & - & 1255.96 & $\delta \mathrm{C}-\mathrm{H}+\nu \mathrm{C}-\mathrm{N}+\nu \mathrm{C}-\mathrm{C}$ \\
\hline 29 & - & 1250 & 1226.74 & $v \mathrm{C}-\mathrm{N}+v \mathrm{C}-\mathrm{C}+\delta \mathrm{C}-\mathrm{H}$ & - & - & 1225.67 & $\delta \mathrm{N}-\mathrm{H}+\delta \mathrm{C}-\mathrm{H}+v \mathrm{C}-\mathrm{N}$ \\
\hline 30 & - & 1225 & 1201.04 & $\delta \mathrm{N}-\mathrm{H}+\delta \mathrm{C}-\mathrm{H}$ & - & - & 1198.25 & $\delta \mathrm{N}-\mathrm{H}+\delta \mathrm{C}-\mathrm{H}$ \\
\hline 31 & 1198 & - & 1169.72 & $v \mathrm{C}-\mathrm{C}+\delta \mathrm{C}-\mathrm{H}$ & 1258 & 1214 & 1173.76 & $\delta \mathrm{C}=\mathrm{C}+\delta \mathrm{N}-\mathrm{H}+\delta \mathrm{C}-\mathrm{H}$ \\
\hline 32 & - & 1184 & 1168.23 & $v \mathrm{C}-\mathrm{C}+\delta \mathrm{C}-\mathrm{H}$ & - & - & 1167.49 & $\delta \mathrm{C}-\mathrm{H}+\delta \mathrm{N}-\mathrm{H}+v \mathrm{C}-\mathrm{C}$ \\
\hline 33 & 1175 & - & 1151.86 & $\delta \mathrm{C}-\mathrm{H}+\delta \mathrm{N}-\mathrm{H}+\nu \mathrm{C}-\mathrm{N}$ & 1209 & - & 1163.41 & $\delta \mathrm{C}-\mathrm{H}+\delta \mathrm{N}-\mathrm{H}+\nu \mathrm{C}-\mathrm{N}$ \\
\hline 34 & - & 1146 & 1134.32 & $\delta \mathrm{C}-\mathrm{H}$ & - & - & 1140.71 & $\delta \mathrm{C}-\mathrm{H}+\delta \mathrm{N}-\mathrm{H}$ \\
\hline 35 & 1129 & - & 1122.09 & $\delta \mathrm{C}-\mathrm{H}+\delta \mathrm{N}-\mathrm{H}+\nu \mathrm{C}-\mathrm{N}$ & 1179 & - & 1132.16 & $\delta \mathrm{C}-\mathrm{H}+\delta \mathrm{N}-\mathrm{H}$ \\
\hline 36 & - & 1097 & 1080.76 & $\delta \mathrm{C}-\mathrm{H}$ & 1144 & - & 1085.63 & $\delta \mathrm{C}-\mathrm{H}+v \mathrm{C}-\mathrm{C}_{\text {ring }}$ \\
\hline 37 & 1098 & - & 1079.58 & $\delta \mathrm{C}-\mathrm{C}+\delta \mathrm{C}-\mathrm{H}$ & 1097 & - & 1079.63 & $\delta \mathrm{C}-\mathrm{H}+v \mathrm{C}-\mathrm{C}_{\text {ring }}$ \\
\hline 38 & 1075 & - & 1051.04 & $v \mathrm{C}-\mathrm{C}+\delta \mathrm{C}-\mathrm{C}+\delta \mathrm{N}-\mathrm{H}$ & 1074 & 1097 & 1012.68 & $\delta \mathrm{C}-\mathrm{H}+v \mathrm{C}-\mathrm{C}_{\text {ring }}$ \\
\hline 39 & - & 1011 & 1002.59 & $\delta \mathrm{C}-\mathrm{H}$ & 1019 & - & 1003.28 & $\delta \mathrm{C}-\mathrm{H}+v \mathrm{C}-\mathrm{C}_{\text {ring }}$ \\
\hline 40 & 1011 & - & 1000.51 & $v \mathrm{C}-\mathrm{C}_{\text {ring }}+\delta \mathrm{C}-\mathrm{H}$ & 1004 & 1006 & 979.67 & $v \mathrm{C}-\mathrm{C}+\delta \mathrm{N}-\mathrm{H}$ \\
\hline 41 & - & - & 958.97 & $\tau \mathrm{C}-\mathrm{H}$ & - & - & 966.82 & $\gamma \mathrm{C}-\mathrm{H}$ \\
\hline 42 & - & - & 958.94 & $\omega \mathrm{C}-\mathrm{H}$ & - & - & 960.63 & $\gamma \mathrm{C}-\mathrm{H}$ \\
\hline 43 & 945 & - & 933.35 & $\omega \mathrm{C}-\mathrm{H}$ & 962 & 962 & 944.80 & $\delta \mathrm{C}-\mathrm{C}+\delta \mathrm{N}-\mathrm{H}+\delta \mathrm{C}-\mathrm{H}$ \\
\hline 44 & - & - & 933.23 & $\tau \mathrm{C}-\mathrm{H}$ & - & - & 934.39 & $\gamma \mathrm{C}-\mathrm{H}$ \\
\hline 45 & - & 939 & 920.76 & $\delta \mathrm{C}-\mathrm{C}+\delta \mathrm{C}-\mathrm{H}$ & 943 & - & 925.97 & $\gamma \mathrm{C}-\mathrm{H}$ \\
\hline
\end{tabular}


Table 3. Cont

\begin{tabular}{|c|c|c|c|c|c|c|c|c|}
\hline \multirow{3}{*}{ Mode } & \multicolumn{4}{|c|}{ Indigo } & \multicolumn{4}{|c|}{ Indirubin } \\
\hline & \multicolumn{2}{|c|}{ Experimental } & \multirow{2}{*}{$\begin{array}{c}\text { Scaled } \\
\text { Computational }\end{array}$} & \multirow{2}{*}{$\begin{array}{c}\text { Tentative } \\
\text { Assignment }{ }^{1}\end{array}$} & \multicolumn{2}{|c|}{ Experimental } & \multirow{2}{*}{$\begin{array}{c}\text { Scaled } \\
\text { Computational }\end{array}$} & \multirow{2}{*}{$\begin{array}{c}\text { Tentative } \\
\text { Assignment }{ }^{1}\end{array}$} \\
\hline & IR & Raman & & & IR & Raman & & \\
\hline 46 & 879 & - & 856.57 & $\delta \mathrm{C}-\mathrm{C}+\delta \mathrm{C}-\mathrm{N}+\delta \mathrm{N}-\mathrm{H}$ & 891 & - & 868.21 & $\delta \mathrm{C}-\mathrm{C}+\delta \mathrm{N}-\mathrm{H}+\delta \mathrm{C}-\mathrm{H}$ \\
\hline 47 & - & 861 & 845.06 & $\delta \mathrm{C}-\mathrm{C}+\delta \mathrm{N}-\mathrm{H}+\delta \mathrm{C}-\mathrm{N}$ & - & - & 855.81 & $\delta \mathrm{C}-\mathrm{C}+\delta \mathrm{N}-\mathrm{H}+\delta \mathrm{C}-\mathrm{H}$ \\
\hline 48 & 858 & - & 841.66 & $\omega \mathrm{C}-\mathrm{H}$ & - & - & 844.28 & $\gamma \mathrm{C}-\mathrm{H}$ \\
\hline 49 & - & - & 840.65 & $\tau \mathrm{C}-\mathrm{H}$ & - & - & 842.74 & $\gamma \mathrm{C}-\mathrm{H}$ \\
\hline 50 & - & - & 790.96 & $\gamma \mathrm{C}-\mathrm{H}+\gamma \mathrm{C}-\mathrm{C}$ & - & - & 794.85 & $\gamma \mathrm{C}-\mathrm{C}+\gamma \mathrm{C}-\mathrm{H}$ \\
\hline 51 & - & - & 781.77 & $\gamma \mathrm{C}-\mathrm{H}+\gamma \mathrm{C}-\mathrm{C}$ & 805 & - & 794.81 & $v \mathrm{C}-\mathrm{C}+\delta \mathrm{C}-\mathrm{C}+\delta \mathrm{C}-\mathrm{H}$ \\
\hline 52 & 790 & - & 754.56 & $\delta \mathrm{C}-\mathrm{C}+\delta \mathrm{C}-\mathrm{H}$ & 783 & - & 764.1 & $\gamma \mathrm{C}=\mathrm{O}+\gamma \mathrm{C}-\mathrm{N}+\gamma \mathrm{C}-\mathrm{H}$ \\
\hline 53 & - & 763 & 748.02 & $\delta \mathrm{C}=\mathrm{C}+\delta \mathrm{C}-\mathrm{N}$ & - & - & 741.01 & $\gamma \mathrm{C}-\mathrm{H}$ \\
\hline 54 & 753 & - & 738.83 & $\omega \mathrm{C}-\mathrm{H}$ & 751 & - & 739.95 & $\gamma \mathrm{C}-\mathrm{H}+\gamma \mathrm{C}-\mathrm{C}_{\text {ring }}$ \\
\hline 55 & - & - & 738.23 & $\tau \mathrm{C}-\mathrm{H}$ & - & - & 729.31 & $\gamma \mathrm{C}-\mathrm{H}+\gamma \mathrm{C}-\mathrm{C}_{\text {ring }}$ \\
\hline 56 & - & - & 709.24 & $\tau \mathrm{C}-\mathrm{H}$ & - & - & 708.19 & $\delta \mathrm{C}-\mathrm{C}+\delta \mathrm{C}-\mathrm{H}+\delta \mathrm{N}-\mathrm{C}$ \\
\hline 57 & 714 & - & 694.24 & $\delta \mathrm{C}-\mathrm{C}+\delta \mathrm{C}-\mathrm{N}$ & 711 & - & 705.46 & $\gamma \mathrm{C}-\mathrm{H}+\gamma \mathrm{N}-\mathrm{H}+\gamma \mathrm{C}-\mathrm{C}$ \\
\hline 58 & 700 & - & 694.01 & $\gamma \mathrm{C}-\mathrm{C}+\gamma \mathrm{C}-\mathrm{H}$ & 682 & 680 & 669.41 & $\delta \mathrm{C}-\mathrm{C}+\delta \mathrm{C}-\mathrm{H}+\delta \mathrm{C}-\mathrm{N}$ \\
\hline 59 & - & 598 & 666.65 & $\delta \mathrm{N}-\mathrm{H}+\delta \mathrm{C}-\mathrm{C}+\delta \mathrm{C}-\mathrm{H}$ & - & 669 & 661.22 & $\delta \mathrm{C}-\mathrm{C}+\delta \mathrm{C}-\mathrm{H}+\delta \mathrm{N}-\mathrm{H}$ \\
\hline 60 & - & - & 591.00 & $\delta \mathrm{N}-\mathrm{H}+\delta \mathrm{C}-\mathrm{C}+\delta \mathrm{C}-\mathrm{H}$ & 632 & 631 & 623.64 & $\delta \mathrm{C}-\mathrm{C}+\delta \mathrm{C}-\mathrm{H}+\delta \mathrm{N}-\mathrm{H}$ \\
\hline 61 & 642 & - & 587.87 & $\delta \mathrm{C}-\mathrm{C}+\delta \mathrm{N}-\mathrm{H}+\delta \mathrm{C}=\mathrm{O}$ & 576 & - & 609.31 & $\gamma \mathrm{N}-\mathrm{H}$ \\
\hline 62 & 596 & - & 553.11 & $\delta \mathrm{C}-\mathrm{C}+\delta \mathrm{C}-\mathrm{H}$ & - & 584 & 572.84 & $\delta \mathrm{C}-\mathrm{C}+\delta \mathrm{N}-\mathrm{H}+\delta \mathrm{C}=\mathrm{O}$ \\
\hline 63 & - & - & 552.34 & $\tau \mathrm{N}-\mathrm{H}+\tau \mathrm{C}-\mathrm{H}$ & - & - & 565.92 & $\delta \mathrm{C}-\mathrm{H}+\delta \mathrm{C}=\mathrm{O}+\delta \mathrm{N}-\mathrm{H}$ \\
\hline 64 & 564 & - & 551.13 & $\omega \mathrm{N}-\mathrm{H}+\omega \mathrm{C}-\mathrm{H}$ & - & - & 556.22 & $\gamma \mathrm{C}-\mathrm{H}+\gamma \mathrm{C}-\mathrm{C}$ \\
\hline 65 & - & 544 & 538.73 & $\delta \mathrm{C}-\mathrm{C}+\delta \mathrm{C}-\mathrm{H}$ & - & - & 540.03 & $\gamma \mathrm{C}-\mathrm{H}+\gamma \mathrm{N}-\mathrm{H}+\gamma \mathrm{C}-\mathrm{C}$ \\
\hline 66 & 524 & - & 504.10 & $\delta \mathrm{N}-\mathrm{H}+\delta \mathrm{C}-\mathrm{C}$ & 536 & 532 & 527.13 & $\delta \mathrm{C}-\mathrm{C}+\delta \mathrm{N}-\mathrm{H}+\delta \mathrm{C}-\mathrm{H}$ \\
\hline 67 & - & - & 472.33 & $\tau \mathrm{N}-\mathrm{H}$ & 497 & 496 & 494.40 & $\gamma \mathrm{N}-\mathrm{H}$ \\
\hline 68 & 509 & - & 465.70 & $\omega \mathrm{N}-\mathrm{H}$ & 471 & 471 & 483.97 & $\delta \mathrm{C}-\mathrm{H}+\delta \mathrm{C}-\mathrm{C}+\delta \mathrm{C}-\mathrm{N}$ \\
\hline 69 & - & 465 & 443.70 & $\gamma \mathrm{C}=\mathrm{C}+\tau \mathrm{C}-\mathrm{H}+\tau \mathrm{N}-\mathrm{H}$ & 450 & - & 449.31 & $\gamma \mathrm{N}-\mathrm{H}+\gamma \mathrm{C}-\mathrm{H}+\gamma \mathrm{C}-\mathrm{C}$ \\
\hline 70 & 420 & - & 418.13 & $\omega \mathrm{C}-\mathrm{H}$ & - & - & 424.65 & $\gamma \mathrm{N}-\mathrm{H}+\gamma \mathrm{C}-\mathrm{H}+\gamma \mathrm{C}-\mathrm{C}$ \\
\hline 71 & - & - & 395.95 & $\tau \mathrm{C}-\mathrm{H}$ & - & - & 398.24 & $\gamma \mathrm{N}-\mathrm{H}+\gamma \mathrm{C}-\mathrm{H}+\gamma \mathrm{C}-\mathrm{C}$ \\
\hline 72 & - & - & 372.22 & $\omega \mathrm{C}-\mathrm{N}+\omega \mathrm{C}-\mathrm{C}$ & - & - & 359.51 & $\delta \mathrm{C}=\mathrm{O}+\delta \mathrm{C}-\mathrm{H}+\delta \mathrm{C}-\mathrm{C}$ \\
\hline 73 & - & 310 & 300.02 & $\delta \mathrm{C}-\mathrm{H}+\delta \mathrm{C}=\mathrm{O}+\delta \mathrm{C}-\mathrm{C}$ & - & - & 342.35 & $\gamma \mathrm{C}-\mathrm{H}+\gamma \mathrm{N}-\mathrm{H}+\gamma \mathrm{C}-\mathrm{C}$ \\
\hline 74 & - & - & 280.89 & $\delta \mathrm{C}-\mathrm{H}+\delta \mathrm{C}=\mathrm{O}+\delta \mathrm{C}-\mathrm{C}$ & - & - & 290.95 & $\delta \mathrm{C}=\mathrm{O}+\delta \mathrm{N}-\mathrm{H}+\delta \mathrm{C}-\mathrm{H}$ \\
\hline 75 & - & 265 & 251.28 & $\tau \mathrm{C}-\mathrm{N}+\tau \mathrm{C}-\mathrm{H}+\tau \mathrm{C}-\mathrm{C}$ & - & - & 268.83 & $\gamma \mathrm{C}-\mathrm{N}+\gamma \mathrm{C}-\mathrm{C}+\gamma \mathrm{C}-\mathrm{H}$ \\
\hline 76 & - & 251 & 242.76 & $\varrho \mathrm{C}-\mathrm{H}$ & - & 210 & 250.91 & $\delta \mathrm{C}=\mathrm{O}+\delta \mathrm{C}-\mathrm{C}$ \\
\hline 77 & - & - & 223.97 & $\omega \mathrm{C}-\mathrm{C}+\omega \mathrm{C}-\mathrm{N}$ & - & - & 235.48 & $\gamma \mathrm{C}-\mathrm{C}+\gamma \mathrm{C}-\mathrm{N}+\gamma \mathrm{C}-\mathrm{H}$ \\
\hline 78 & - & 172 & 222.05 & $\delta \mathrm{C}=\mathrm{O}+\delta \mathrm{C}-\mathrm{H}+\delta \mathrm{N}-\mathrm{H}$ & - & - & 215.76 & $\delta \mathrm{C}-\mathrm{H}$ \\
\hline 79 & - & - & 155.98 & $\omega \mathrm{C}=\mathrm{O}+\omega \mathrm{C}-\mathrm{H}$ & - & - & 144.67 & $\gamma \mathrm{C}-\mathrm{H}+\gamma \mathrm{N}-\mathrm{C}+\gamma \mathrm{C}=\mathrm{O}$ \\
\hline 80 & - & 137 & 153.90 & $\tau \mathrm{C}=\mathrm{O}+\tau \mathrm{C}-\mathrm{H}$ & - & 116 & 139.47 & $\gamma \mathrm{C}-\mathrm{H}+\gamma \mathrm{N}-\mathrm{C}+\gamma \mathrm{C}=\mathrm{O}$ \\
\hline 81 & - & - & 93.86 & $\tau \mathrm{C}-\mathrm{H}+\tau$ ring & - & - & 108.76 & $\gamma \mathrm{C}-\mathrm{H}+\gamma \mathrm{C}=\mathrm{O}$ \\
\hline 82 & - & - & 81.45 & $\tau$ ring & - & - & 103.18 & $\delta \mathrm{C}-\mathrm{H}$ \\
\hline 83 & - & - & 67.99 & $\delta \mathrm{C}-\mathrm{H}+\delta \mathrm{N}-\mathrm{H}$ & - & - & 61.04 & $\tau$ ring \\
\hline 84 & - & - & 29.29 & $\omega$ ring & - & - & 38.09 & $\omega$ ring \\
\hline
\end{tabular}

${ }^{1} v$ : stretching; $v_{\mathrm{s}}$ : symmetrical stretching; $v_{\text {as }}$ : asymmetrical stretching; $\delta$ : in-plane deformation; $\gamma$ : out-of-plane deformation; $\varrho$ : rocking; $\omega$ : wagging; $\tau$ : twisting.

As can be seen from Table 3, slight discrepancies between the measured and simulated wavenumbers are attributed to the computational system that only a single molecule in the gas phase was adopted by neglecting inter-molecular interactions. There are 30 atoms and 84 fundamental vibrations in both indigo and indirubin molecules. Indigo belongs to the $C_{2 h}$ group with a center of symmetry, which holds the mutual exclusion rule that only the gerade vibrations are Raman active and the ungerade vibrations are IR active. On the contrary, there is no mutual exclusion rule in the polar indirubin molecule.

The experimental vibrational spectra and assignments of indigo are presented in Figure 5 and Table 3. The broad band observed at $3436 \mathrm{~cm}^{-1}$ in the FTIR spectrum is assigned to the stretching vibration of the N-H bond. The strongest intensity in IR band located at $3269 \mathrm{~cm}^{-1}$ is assigned to the stretching vibration of the chelation structure of $\mathrm{C}=\mathrm{O}$ and hydrogen bonds. The stretching vibration of $\mathrm{C}-\mathrm{H}$ in the aromatic ring is between 2853 and $3060 \mathrm{~cm}^{-1}$ in the IR spectrum. The spectral range of $1586-1701 \mathrm{~cm}^{-1}$ is assigned to the stretching vibration of the conjugated system of $\mathrm{C}=\mathrm{C}, \mathrm{C}=\mathrm{O}$, and $\mathrm{N}-\mathrm{H}$ groups, and this range is in response to indigoid molecules $[19,29]$. The ring $C-C$ stretching bands also appeared in the FTIR and Raman spectrum at 1483 and $1462 \mathrm{~cm}^{-1}$. The region below $1394 \mathrm{~cm}^{-1}$ can be mainly ascribed to the deformation vibrations of $\mathrm{N}-\mathrm{H}$ and $\mathrm{C}-\mathrm{H}$.

The experimental vibrational spectra and assignments of indirubin are also given in Figure 5 and Table 3. The strongest intensity IR band located at $3433 \mathrm{~cm}^{-1}$ is assigned to the stretching vibration of the $\mathrm{N}-\mathrm{H}$ bond. The stretching vibration of the chelation structure of $\mathrm{C}=\mathrm{O}$ and hydrogen bond appears at $3346 \mathrm{~cm}^{-1}$ in the FTIR spectrum. The spectral range of $3230-2853 \mathrm{~cm}^{-1}$ is corresponding to the stretching vibration of $\mathrm{C}-\mathrm{H}$ in the aromatic ring. The stretching vibration of the conjugated system 
of $\mathrm{C}=\mathrm{C}, \mathrm{C}=\mathrm{O}$, and $\mathrm{N}-\mathrm{H}$ groups is observed between 1587 and $1703 \mathrm{~cm}^{-1}$. The ring $\mathrm{C}-\mathrm{C}$ stretching bands are also observed at 1482 and $1465 \mathrm{~cm}^{-1}$ in IR (1479 and $1460 \mathrm{~cm}^{-1}$ in Raman). The deformation vibrations of $\mathrm{N}-\mathrm{H}$ and $\mathrm{C}-\mathrm{H}$ can be assigned below $1385 \mathrm{~cm}^{-1}$.

The assignments show that the corresponding vibrational modes in indigo and indirubin present different wavenumbers and Raman shifts, indicating their different symmetries. The strongest intensities of indigo are at $3269 \mathrm{~cm}^{-1}$ (FTIR) and at $1572 \mathrm{~cm}^{-1}$ (Raman), while those of indirubin are located at $3433 \mathrm{~cm}^{-1}$ and $1587 \mathrm{~cm}^{-1}$, respectively. The same vibrational mode presents a higher frequency in indirubin than that in indigo because indirubin is more polar and less conjugated. The intermolecular hydrogen bonds in indigo and indirubin are two and one, respectively, so the IR band located at $3269 \mathrm{~cm}^{-1}$ in indigo is wider than the IR band located at $3346 \mathrm{~cm}^{-1}$ in indirubin, because of stretching vibration of chelation structure of $\mathrm{C}=\mathrm{O}$ and hydrogen bonds.

\subsection{UV-Visible Spectra Analysis}

The UV-Visible absorption spectra of indigo and indirubin obtained in the experiments are plotted in Figure 6. Both experimental and computational absorption wavelengths, transition coefficient, oscillator strengths, and assignments for indigo and indirubin are detailed in Table 4.

(a)

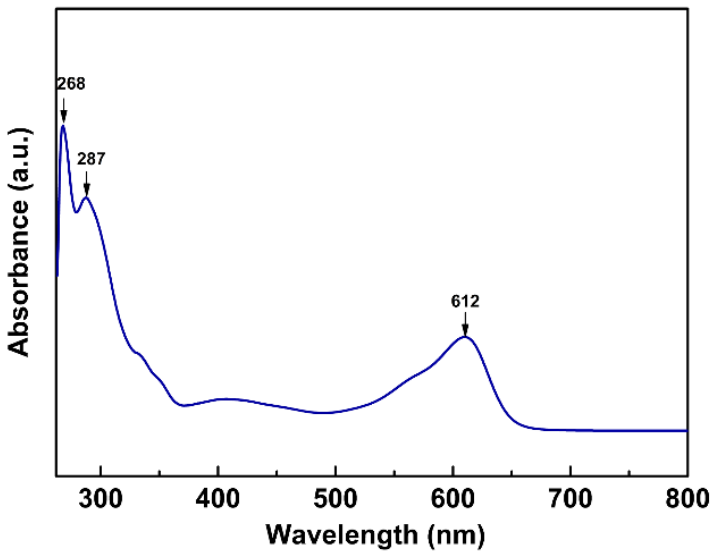

(b)

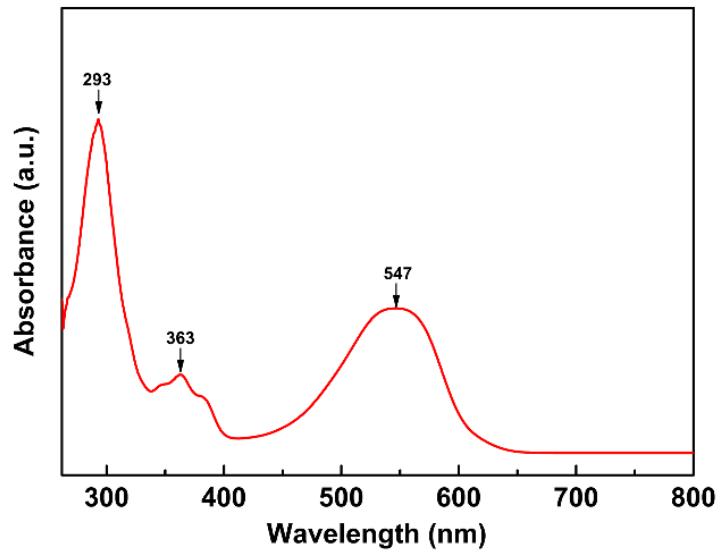

Figure 6. UV-Visible absorption spectra of indigo (a) and indirubin (b).

Table 4. Theoretical UV-Visible absorption spectra of indigo and indirubin computational at B3LYP/6-311G $(\mathrm{d}, \mathrm{p})$ level of theory.

\begin{tabular}{|c|c|c|c|c|c|}
\hline \multirow{2}{*}{ Molecular } & \multicolumn{2}{|c|}{ Wavelength (nm) } & \multirow{2}{*}{$\begin{array}{l}\text { Transition } \\
\text { Coefficient }\end{array}$} & \multirow{2}{*}{$\begin{array}{c}\text { Oscillator } \\
\text { Strengths (f) }\end{array}$} & \multirow{2}{*}{ Assignment $^{1}$} \\
\hline & Experimental & Computational & & & \\
\hline \multirow[t]{2}{*}{ Indigo } & 612 & 579.77 & 0.70811 & 0.3342 & $\mathrm{H} \rightarrow \mathrm{L}$ \\
\hline & 268 & 272.14 & 0.66336 & 0.5277 & $\mathrm{H}-1 \rightarrow \mathrm{L}+1$ \\
\hline \multirow[t]{2}{*}{ Indirubin } & 547 & 536.36 & 0.70152 & 0.2591 & $\mathrm{H} \rightarrow \mathrm{L}$ \\
\hline & 293 & 259.85 & 0.52164 & 0.1432 & $\mathrm{H}-1 \rightarrow \mathrm{L}+1$ \\
\hline
\end{tabular}

${ }^{1} \mathrm{H}=$ highest occupied molecular orbital; $\mathrm{L}=$ lowest unoccupied molecular orbital.

As can be seen from Table 4 that indigo has two characteristic absorption peaks at 268 and $287 \mathrm{~nm}$ in the ultraviolet region and one characteristic absorption peak at $612 \mathrm{~nm}$ in the visible light region. Indirubin has two characteristic absorption peaks at 293 and $363 \mathrm{~nm}$ in the ultraviolet region and one characteristic absorption peak at $547 \mathrm{~nm}$ in the visible light region. The maximum absorption wavelengths of indigo and indirubin are 612 and $547 \mathrm{~nm}$, respectively, both of which are higher than the computational values. This may be due to neglecting intermolecular interactions between molecules and solvents in the calculations. Indigo and indirubin molecules are large conjugated systems, which results in a broad absorption peak at 612 and $547 \mathrm{~nm}$, and the maximum absorption 
wavelength generated a bathochromic shift. As reported before, the maximum absorption at 612 and $547 \mathrm{~nm}$ are assigned to the transition from HOMO to LUMO orbital, which has a $\pi \rightarrow \pi^{*}$ character [29]. Due to the smaller degree of conjugation of indirubin, its maximum absorption wavelength is shorter. The strongest absorption bands at $268 \mathrm{~nm}$ (indigo) and $293 \mathrm{~nm}$ (indirubin) are signed to HOMO-1 $\rightarrow$ LUMO +1 transition that also has a $\pi \rightarrow \pi^{*}$ feature. This difference might be because the positions of the carbonyl and the imino group in the two molecules are different, resulting in different degrees of steric hindrance effect in the molecules. Consequently, the planar properties, conjugated degree, and absorption wavelength of the two molecules are different.

\subsection{Fluorescence Spectra Analysis}

The electrons in the $\pi^{*}$ orbit are unstable and quickly return to the ground state, thereby generating fluorescence. The first singlet excited state generated a $\pi \rightarrow \pi^{*}$ transition when absorbing energy, because of their large degree of conjugation. The fluorescence spectra of indigo and indirubin were obtained in the experiments as shown in Figure 7. Experimental and computational emission wavelengths, transition coefficient, oscillator strengths, and assignments for indigo and indirubin are detailed in Table 5 .

(a)

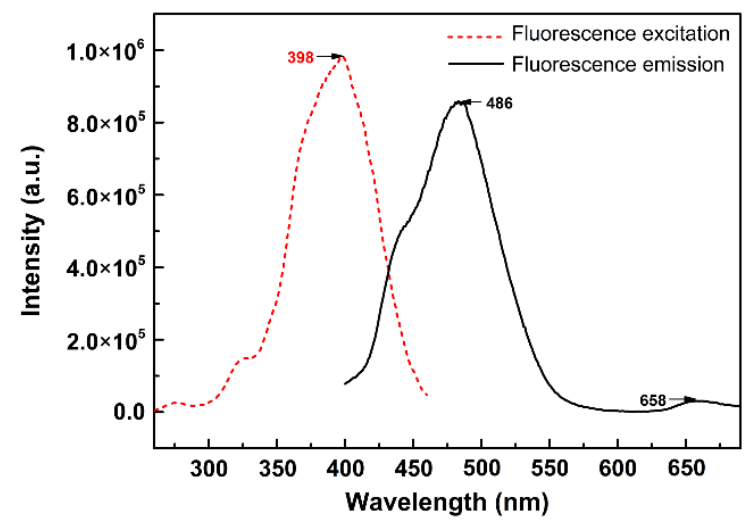

(b)

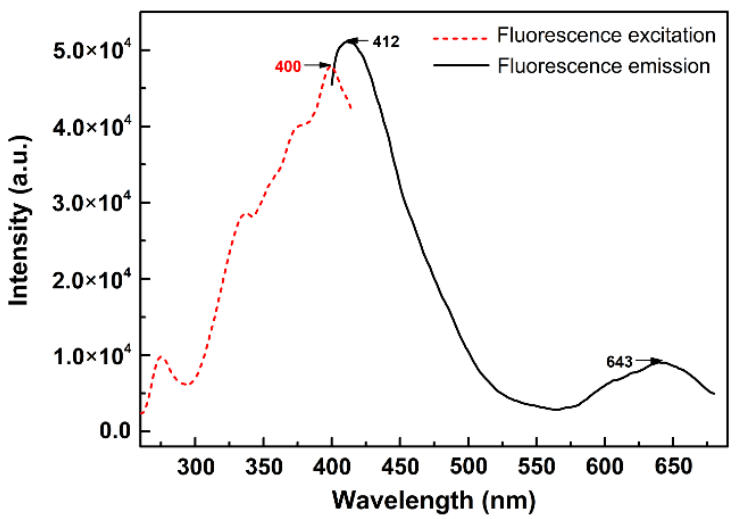

Figure 7. Fluorescence spectra of indigo (a) and indirubin (b).

Table 5. Theoretical electronic emission spectra of indigo and indirubin computational at the B3LYP/6-311G(d,p) level of theory.

\begin{tabular}{|c|c|c|c|c|c|}
\hline \multirow{2}{*}{ Molecular } & \multicolumn{2}{|c|}{ Wavelength (nm) } & \multirow{2}{*}{$\begin{array}{l}\text { Transition } \\
\text { Coefficient }\end{array}$} & \multirow{2}{*}{$\begin{array}{c}\text { Oscillator } \\
\text { Strengths (f) }\end{array}$} & \multirow{2}{*}{ Assignment ${ }^{1}$} \\
\hline & Experimental & Computational & & & \\
\hline Indigo & $486\left(8.57 \times 10^{5}\right)$ & 438.35 & 0.68114 & 0.7352 & $\mathrm{~L} \rightarrow \mathrm{H}$ \\
\hline Indirubin & $412\left(5.10 \times 10^{4}\right)$ & 433.26 & 0.67598 & 0.6871 & $\mathrm{~L} \rightarrow \mathrm{H}$ \\
\hline
\end{tabular}

${ }^{1} \mathrm{H}=$ highest occupied molecular orbital; $\mathrm{L}=$ lowest unoccupied molecular orbital.

The experimental results in Table 5 show that the strongest fluorescence peak wavelengths of indigo and indirubin are 486 and $412 \mathrm{~nm}$, respectively, both of which are mainly generated by excitation from LUMO $\rightarrow$ HOMO. The difference in peak wavelength is ascribed to that indigo molecules in the excited state have better planar conformation than indirubin which can reduce the molecular vibration, so that the excitation energy of the molecules is not easily released by thermal energy due to vibration. This is beneficial to the generation of fluorescence. Additionally, indigo molecules have a large degree of conjugation, so its fluorescence wavelength is longer, and the peak intensity is also significantly higher than those of indirubin. The computational values agree well with the experimental results, proving that the ground and excited state configuration of the optimized molecule are acceptable. 


\section{Experimental}

\subsection{Materials}

Indigo (98\%) and indirubin $(97.5 \%)$ extracted from Folium Isatidis were purchased from Chengdu PureChem-Standard Co., Ltd. (Chengdu, China) and used as received. The solvent used, N,N-dimethylformamide (DMF, 99.5\%) of analytical grade, was obtained from Sinopharm Chemical Reagent Co., Ltd. (Shanghai, China). Two $100 \mathrm{~mL}$ volumetric flasks with $5 \mathrm{mg}$ indigo and $5 \mathrm{mg}$ indirubin were prepared, and DMF was added to the marks of the two flasks, tightly covered with parafilm, and placed in an ultrasonic bath for $30 \mathrm{~min}$. The solutions of indigo and indirubin of $100 \mu \mathrm{g} \mathrm{ml}^{-1}$ in DMF were obtained.

\subsection{Instrumentation}

\subsubsection{Infrared Spectroscopy}

The room temperature FTIR spectra of indigo and indirubin in the frequency ranging from 4000 to $400 \mathrm{~cm}^{-1}$ were recorded on a Fourier Infrared Raman spectrometer (FTIR, NEXUS-670, Nicolet, Madison, WI, USA) by using a potassium bromide tableting method. The background and samples were scanned 16 times with a resolution of $4 \mathrm{~cm}^{-1}$.

\subsubsection{Raman Spectroscopy}

The Raman spectra of indigo and indirubin at room temperature were measured in the 1800 and $100 \mathrm{~cm}^{-1}$ intervals with an InVia Reflex Micro Raman Spectrometer (Renishaw, Gloucester, UK). The Raman data were collected by using the Ar laser of $532 \mathrm{~nm}$ and a 50× objective.

\subsubsection{UV-Visible Spectroscopy}

UV-visible spectra measurements were recorded on a Lambda 35 UV-Visible NIR spectrophotometer (PerkinElmer, Waltham, MA, USA) using a quartz cuvette. The solutions of indigo and indirubin in DMF were prepared for this test. The spectra were recorded between 265 and $750 \mathrm{~nm}$.

\subsubsection{Fluorescence Spectrometry}

Fluorescence spectra were recorded using a quartz cuvette on the QM/TM steady state/transient fluorescence spectrometer (PTI, Birmingham, NJ, USA). All slit widths were set to $5 \mathrm{~nm}$, and the emission wavelength of $480 \mathrm{~nm}$ was selected for the $100 \mu \mathrm{g} \mathrm{ml}^{-1}$ indigo/DMF solution. The excitation spectrum was recorded between 260 and $460 \mathrm{~nm}$, from which the optimal excitation wavelength was selected. The emission spectrum between $400 \mathrm{~nm}$ and $680 \mathrm{~nm}$ was recorded at $380 \mathrm{~nm}$. Under the same conditions, the excitation spectrum of the $100 \mu \mathrm{g} \mathrm{ml}^{-1}$ indirubin/DMF solution was measured, and the optimum excitation wavelength was set as $350 \mathrm{~nm}$. Its emission spectrum between $370 \mathrm{~nm}$ and $680 \mathrm{~nm}$ was recorded.

\subsection{Computational Details}

With the use of the density functional theory (DFT) method, the optimized geometric structures, vibrational frequency, molecular electrostatic potential, and frontier molecular orbitals of indigo and indirubin were calculated at Becke3-Lee-Yang-Parr (B3LYP) with 6-311G(d,p) basis set. The single-excitation configuration interaction (CIS) was used to optimize the excited state structure. Based on the optimized structure, the time-dependent density functional theory (TD-DFT) and the polarization continuum model (PCM) were used together to calculate the absorption spectra of the two molecules in the ground state configuration and the fluorescence spectra in the excited state configuration at B3LYP with 6-311G(d,p). All the calculations were performed using the program 
package Gaussian 16 [30]. Gauss view 6 was used to build Gaussian input files and visualize the results of the Gaussian calculations [31].

\section{Conclusions}

The molecular structures and spectral properties of indigo and indirubin from natural resources have been characterized experimentally and computationally. The steric hindrance effect, plane and conjugated degree of the two molecules were different, giving rise to different spectral properties. The main conclusions can be drawn as below.

(1) It is confirmed that both indigo and indirubin molecules form a large stable conjugated system. Indirubin has a higher frontier orbital energy gap and better chemical stability than indigo.

(2) The strongest intensities of indigo are at $3269 \mathrm{~cm}^{-1}$ (FTIR) and at $1572 \mathrm{~cm}^{-1}$ (Raman), but those of indirubin are located at $3433 \mathrm{~cm}^{-1}$ and $1587 \mathrm{~cm}^{-1}$, respectively.

(3) The main absorption bands of indigo and indirubin are mainly assigned to the transition from $\mathrm{HOMO} \rightarrow$ LUMO orbital and HOMO- $\rightarrow$ LUMO+1 orbital, which have a $\pi \rightarrow \pi^{*}$ character. Indirubin has lower planeness, smaller degree of conjugation than indigo, thereby having smaller maximum absorption wavelength.

(4) The fluorescence wavelength of indigo is longer, and the peak intensity is also significantly higher than those of indirubin. The strongest fluorescence peak wavelengths of indigo and indirubin are mainly generated by excitation from LUMO $\rightarrow \mathrm{HOMO}$, which have a $\pi \rightarrow \pi^{*}$ character. Indigo molecules in the excited state have better planar properties and conjugation, which can reduce molecular vibrations, so the excitation energy of the molecules is not easily released by thermal energy due to vibration. This is the reason why indigo generates strong fluorescence.

The vibrational spectra of indigo and indirubin are different in various wavelengths, so synthetic indigo without any impurities could have different features compared with natural indigo containing the main impurity, indirubin. This provides a practicable way to distinguish natural and synthetic indigo by using vibrational spectra.

Supplementary Materials: The following are available online, Figure S1: The correlation between computational and literature data of bond lengths and angles.

Author Contributions: Conceptualization, Y.L.; methodology, Y.L. and Z.J.; software, Z.J.; investigation, Y.L., J.S. and Z.J.; resources, J.S.; data curation, Z.J.; writing—original draft preparation, Z.J.; writing-review and editing, Y.L. and Z.J.; supervision, Y.L. and J.S.; funding acquisition, J.S.

Funding: This research was funded by the Shanghai Naturalism Biological Technology Co., Ltd. (grant number 10117517).

Conflicts of Interest: The authors declare no conflict of interest.

\section{References}

1. Namgung, S.; Park, H.A.; Kim, J.; Lee, P.G.; Kim, B.G.; Yang, Y.H.; Choi, K.Y. Ecofriendly one-pot biosynthesis of indigo derivative dyes using CYP102G4 and PrnA halogenase. Dyes Pigment. 2019, 162, 80-88. [CrossRef]

2. He, B.; Pun, A.B.; Zherebetskyy, D.; Liu, Y.; Liu, F.; Klivansky, L.M.; McGough, A.M.; Zhang, B.A.; Lo, K.; Russell, T.P.; et al. New form of an old natural dye: Bay-annulated indigo (BAI) as an excellent electron accepting unit for high performance organic semiconductors. J. Am. Chem. Soc. 2014, 136, 15093-15101. [CrossRef] [PubMed]

3. Serafini, I.; Lombardi, L.; Fasolato, C.; Sergi, M.; Di, O.F.; Sciubba, F.; Montesano, C.; Guiso, M.; Costanza, R.; Nucci, L. A new multi analytical approach for the identification of synthetic and natural dyes mixtures. The case of orcein-mauveine mixture in a historical dress of a Sicilian noblewoman of nineteenth century. Nat. Prod. Res. 2017, 1-12. [CrossRef] [PubMed]

4. Süsse, P.; Steins, M.; Kupcik, V. Indigo: Crystal structure refinement based on synchrotron data. Z. Krist.-Cryst. Mater. 1988, 184, 269-273. [CrossRef]

5. Christie, R.M. Indirubin: The Red Shade of Indigo, 1st ed.; Life in Progress Editions: Roscoff, France, 2006; pp. 103-108. 
6. Lü, H.; Liu, J.; Deng, R.; Song, J. Preparative isolation and purification of indigo and indirubin from folium isatidis by high-speed counter-current chromatography. Phytochem. Anal. 2012, 23, 637-641. [CrossRef] [PubMed]

7. Meksi, N.; Kechida, M.; Mhenni, F. Cotton dyeing by indigo with the borohydride process: Effect of some experimental conditions on indigo reduction and dyeing quality. Chem. Eng. J. 2007, 131, 187-193. [CrossRef]

8. Degani, L.; Riedo, C.; Chiantore, O. Identification of natural indigo in historical textiles by GC-MS. Anal. Bioanal. Chem. 2015, 407, 1695-1704. [CrossRef]

9. Pawlak, K.; Puchalska, M.; Miszczak, A.; Roså,Oniec, E.; Jarosz, M. Blue natural organic dyestuffs-from textile dyeing to mural painting. Separation and characterization of coloring matters present in elderberry, logwood and indigo. J. Mass Spectrom. 2006, 41, 613-622. [CrossRef]

10. Irimia-Vladu, M.; Glowacki, E.D.; Troshin, P.A.; Schwabegger, G.; Leonat, L.; Susarova, D.K.; Krystal, O.; Ullah, M.; Kanbur, Y.; Bodea, M.A.; et al. Indigo-a natural pigment for high performance ambipolar organic field effect transistors and circuits. Adv. Mater. 2012, 24, 375-380. [CrossRef]

11. Tomkinson, J.; Bacci, M.; Picollo, M.; Colognesi, D. The vibrational spectroscopy of indigo: A reassessment. Vib. Spectrosc. 2009, 50, 268-276. [CrossRef]

12. Xue, Y.; Gong, X.; Xiao, H.; Tian, H. A density functional theory study of indigo and its derivatives. Acta Chim. Sinica 2004, 62, 963-968.

13. Cervantes-Navarro, F.; Glossman-Mitnik, D. DFT study of the effect of substituents on the absorption and emission spectra of indigo. Chem. Cent. J. 2012, 6, 70-75. [CrossRef] [PubMed]

14. Jacquemin, D.; Preat, J.; Wathelet, V.; Perpete, E.A. Substitution and chemical environment effects on the absorption spectrum of indigo. J. Chem. Phys. 2006, 124, 74104. [CrossRef] [PubMed]

15. Tatsch, E.; Schrader, B. Near-infrared fourier transform Raman spectroscopy of indigoids. J. Raman Spectrosc. 1995, 26, 467-473. [CrossRef]

16. Rio, M.S.D.; Picquart, M.; Haro-Poniatowski, E.; Elslande, E.V.; Uc, V.H. On the Raman spectrum of Maya blue. J. Raman Spectrosc. 2010, 37, 1046-1053.

17. Ricci, M.; Lofrumento, C.; Becucci, M.; Castellucci, E.M. The Raman and SERS spectra of indigo and indigo-Ag2 complex: DFT calculation and comparison with experiment. Spectroc. Acta Pt. A-Molec. Biomolec. Spectr. 2018, 188, 141-148. [CrossRef]

18. El-Mansy, M.A.M.; Yahia, I.S.; Alfaify, S. Conformational and vibrational properties of indigo dye: DFT approach. Org. Opto-Elect. 2015, 1, 39-45.

19. Baran, A.; Fiedler, A.; Schulz, H.; Baranska, M. In situ Raman and IR spectroscopic analysis of indigo dye. Anal. Methods 2010, 2, 1372-1376. [CrossRef]

20. Rondao, R.; Seixas de Melo, J.; Schaberle, F.A.; Voss, G. Excited state characterization of a polymeric indigo. Phys. Chem. Chem. Phys. 2012, 14, 1778-1783. [CrossRef]

21. Sele, A.; Bremner, J.B.; Willis, A.C.; Haritakun, R.; Grifth, R.; Keller, P.A. A cascade synthetic route to new bioactive spiroindolinepyrido [1,2-a]indolediones from indirubin. Tetrahedron 2016, 47, 8357-8367. [CrossRef]

22. Wang, Y.; Liu, Y.; Yang, M.; Liang, X. Study on the quantum chemistry of indigo and indirubin. J. Sichuan Univ. 2004, 41, 143-147.

23. Xue, Y.; Peng, Z.; Yin, X. DFT study on structure activity relationship for indirubin and its isomer. Chem. Res. 2005, 16, 85-87.

24. Perpète, E.A.; Preat, J.; André, J.-M.; Jacquemin, D. An ab initio study of the absorption spectra of indirubin, isoindigo, and related derivatives. J. Phys. Chem. A 2006, 110, 5629-5635. [CrossRef] [PubMed]

25. Prandraud, H. Structure cristalline de l'indirubine. J. Acta Cryst. 1961, 14, 901-908. [CrossRef]

26. Lu, T.; Chen, F. Multiwfn: A multifunctional wavefunction analyzer. J. Comb. Chem. 2012, 33, 580-592. [CrossRef]

27. Humphrey, W.; Dalke, A.; Schulten, K. VMD: Visual molecular dynamics. J. Mol. Graph. 1996, 14, $33-38$. [CrossRef]

28. Amat, A.; Rosi, F.; Miliani, C.; Sgamellotti, A.; Fantacci, S. Theoretical and experimental investigation on the spectroscopic properties of indigo dye. J. Mol. Struct. 2011, 993, 43-51. [CrossRef]

29. Platania, E.; Lofrumento, C.; Lottini, E.; Azzaro, E.; Ricci, M.; Becucci, M. Tailored micro-extraction method for Raman/SERS detection of indigoids in ancient textiles. Anal. Bioanal. Chem. 2015, 407, 6505-6514. [CrossRef] 
30. Frisch, M.J.; Trucks, G.W.; Schlegel, H.B.; Scuseria, G.E.; Robb, M.A.; Cheeseman, J.R.; Scalmani, G.; Barone, V.; Petersson, G.A.; Nakatsuji, H.; et al. Gaussian 16; Revision, B.01; Gaussian Inc.: Wallingford, CT, USA, 2016.

31. Dennington, R.; Keith, T.A.; Millam, J.M. GaussView, Version 6; Gaussian Inc.: Wallingford, CT, USA, 2016.

Sample Availability: Samples of the compounds are not available from the authors.

(C) 2019 by the authors. Licensee MDPI, Basel, Switzerland. This article is an open access article distributed under the terms and conditions of the Creative Commons Attribution (CC BY) license (http://creativecommons.org/licenses/by/4.0/). 